\section{Vertebral artery dissection stroke in evolution presented with postural headache as initial manifestation}

\author{
Yen-Chung Chen, ${ }^{1}$ Yang-Hao Ou, ${ }^{1}$ \\ Ming-Che Chang, ${ }^{2,3}$ Wei-Liang, Chen, ${ }^{4}$ \\ Chih-Ming Lin1,5 \\ 1Department of Neurology, Changhua \\ Christian Hospital; 2Department of \\ Nuclear Medicine, Changhua Christian \\ Hospital; 3Department of Medical \\ Imaging and Radiological Sciences, \\ Central Taiwan University of Science \\ and Technology, Taichung; ${ }^{4}$ Department \\ of Medical Imaging, Changhua \\ Christian Hospital; 5Department of \\ Social Work and Child Welfare, \\ Providence University, Taichung, Taiwan
}

\section{Abstract}

In young adult, the most common etiology of acute ischemic brain infarction are arterial dissections and cardiogenic embolic stroke. Vertebral artery dissection without preceding trauma history is quite rare in young ischemic stroke patients. Postural headache is even more atypical presentation for vertebral artery dissection. It is often misdiagnosed as spontaneous intracranial hypotension. We described a 37-year-old male suffering from acute onset postural headache with stroke in evolution during hospitalization. The initial brain magnetic resonance imaging (MRI) mislead to diagnosis of ischemic lesion. Nevertheless, with the aid of single photon emission computed tomography, we are confident the patient was afflicted with ischemic/hemorrhagic lesion, instead of neoplasm or demyelinating diseases. Lateral medullary syndrome was confirmed on the repeated brain MRI. His general condition improved with steady gait and clear articulation without easychoking after adequate hydration and rehabilitation training with aspirin as secondary prevention. Cranial artery dissections is a crucial differential diagnosis while thunderclap headache happens even related to postural change without obvious neurological deficit in the beginning presentations.

\section{Introduction}

Dissection of vertebral artery is an uncommon cause of all ischemic strokes with annual incidence estimated at 1 to 1.5 per $100,000.1$ However, it attributes to approximately 1 in 10 ischemic stroke in young patients. 1 Clinical presentation of vertebral artery dissection takes a plethora of courses, depend upon the location of the dissection; it ranges from asymptomatic occlusions, transient ischemic attacks to lateral or medial medullary syndromes. The most common chief complaint is head and/or neck pain, which is found in up to 90 percent of patients. ${ }^{2}$ The headache is typically described as gradual onset that is mostly unilateral and involves the frontotemporal region, ${ }^{3}$ accompanied by vertigo, dizziness, or gait disturbances.

On the other hand, spontaneous intracranial hypotension commonly present with a gradual onset orthostatic headache near the occipital regions. ${ }^{4}$ The leakage of cerebrospinal fluid causes the downward displacement of the brain inducing headache that worsens while standing up and relieves on decumbency.

This case is a young male patient, who suffered from spontaneous dissection of V3 vertebral artery with an initial presentation of acute onset postural headache that mimics the symptom of spontaneous intracranial hypotension.

\section{Case Report}

The 37-year-old Taiwanese male patient presented to our medical attention with acute onset postural headache over right temporal area associating with occasional dizziness. He was well-being prior to this attack and led a normal living as a logistic bus driver for more than nine year. $\mathrm{He}$ claimed to carry heavy object day to day but experienced no particular physical discomfort. The characteristic of headache was worsen while he was standing up shifty and the pain could be partially relieved when he lied down on the back. On reviewing his medical history, he denies any trauma history, fever, neck pain, double vision, blurred vision, weakness or numbness of limbs, fecal or urine incontinence, loss of consciousness before, during or after the attack.

He was therefore admitted to our neurological ward for advanced study. On detailed neurological examination, it revealed he suffered from aggravating dizziness, accompanying with nausea and vomiting several times and unsteady gait leaning to right side upon standing up. Though his vital signs were stable that was checked in the emergency room along with laboratory survey showing insignificant findings, Physical examination demonstrated right facial and tongue paresthesia, mild right central facial palsy, deviation of
Correspondence: Chih-Ming Lin, Department of Neurology, Changhua Christian Hospital, 135 Nanxiao St., Changhua City, Changhua County 500, Taiwan.

Tel.: +886.4.7238595 ext. 4903 .

E-mail:166110@cch.org.tw

Key words: magnetic resonance imaging, single photon emission computed tomography, lateral medullary syndrome, aspirin, headache.

Contributions: $\mathrm{YCC}$ and $\mathrm{YHO}$ contributed equally.

Conflict of interest: the authors declare no potential conflict of interest.

Funding: none.

Received for publication: 28 March 2018 Accepted for publication: 7 May 2018

This work is licensed under a Creative Commons Attribution NonCommercial 4.0 License (CC BY-NC 4.0).

C) Copyright Y-C. Chen et al., 2018

Licensee PAGEPress, Italy

Neurology International 2018; 10:7694

doi:10.4081/ni.2018.7694

tongue toward right side on protrusion and left deviation of uvula with dysarthria without dysphagia on the second day of admission. In addition, his muscle strength (muscle power) and deep tendon reflex tests were both normal; Nevertheless, patient showed significant right-sided dysmetria with abnormal finger-nose test and heelshin test. Right side mild dysdiadochokinesia was also founded. Additionally, rebound phenomenon of right arm and Romberg's sign were also observed.

Initial evaluation with non-contrast cerebral computed tomography showed no intracranial hemorrhage or mass lesions. Brain magnetic resonance imaging showed presence of small focal area of high signal intensity over diffusion weighted sequence at the right cerebellum. The demyelinating disease was suspected and cerebral spinal fluid analysis was done. However, the result showed insignificant findings. To clarify the etiology we arranged single-photon emission computed tomography (SPECT) (Figure 1). It elucidated the lesion was of arteriosus ischemic origin.

During the $5^{\text {th }}$ day of hospitalization, ostensible Horner's syndrome manifested. The cerebral computed tomographic angiography and MRI were repeated and it revealed intracranial vessel wall $3^{\text {rd }}$ portion of vertebral artery dissection (Figure 2) 
along with acute lacunar infarct of the right lateral medulla.

Carotid duplex and transcranial color coded sonography showed increased resistance over the right vertebral artery without obvious end diastolic velocity suggesting upstream critical stenosis and trivial flow over right side intracranial vertebral artery.

After adequate hydration and rehabilitation training with aspirin as secondary prevention, his general condition improved with steady gait and clear articulation without easy-choking. He was discharged and followed at the neurologic clinic up to date.

\section{Discussion}

Vertebral artery dissection is the separation of arterial wall layers. Typically, there is a remote history of trauma of the neck either sustained during sporting events or chiropractic manipulations. The consequences of arterial wall dissection can either reduce the distal blood flow due to sequestration by the false lumen or formation of thromboembolism because the turbulent blood flow. In either case, posterior cerebral blood flow is decreased; most commonly effected organs are cerebellum, and posterior or lateral medulla. Initially, patients with vertebral artery dissection complains of headache or neck pain, as the disease progresses, patient may experience vertigo, ataxia, impaired fine movement coordination, abnormal sensation of the face and trunk, dysphasia, or Horner syndrome. Given the delayed onset of neurological symptoms, vertebral artery dissection is usually not diagnosed until later during hospitalization. ${ }^{5}$ While this patient did not have significant history of trauma that warrants for investigation of vertebral artery dissection; but arriving in the emergency room with new onset of headache should raise red flag to any clinicians. Orthostatic headache has been classically associated with intracranial hypotension due to CSF leak. Even though, its incidence is only approximately $1 \%$ of all emergency room headaches, ${ }^{6}$ nonetheless, it is still an important differential diagnosis to entertain. Given patient's initial presentation and inconclusive brain MRI, he was admitted with tentative diagnosis of spontaneous intracranial hypotension.

Initial brain MRI showed a focal lesion over right cerebellum with hyperintensity on DWI, ADC, T2-FLAIR and TI weighted sequence. After discussion with the radiologist, subacute hemorrhage or acute infarction with hemorrhagic transformation or demyelinating diseases are in the differential diagnosis. Therefore, $99 \mathrm{mTc}-\mathrm{ECD}$

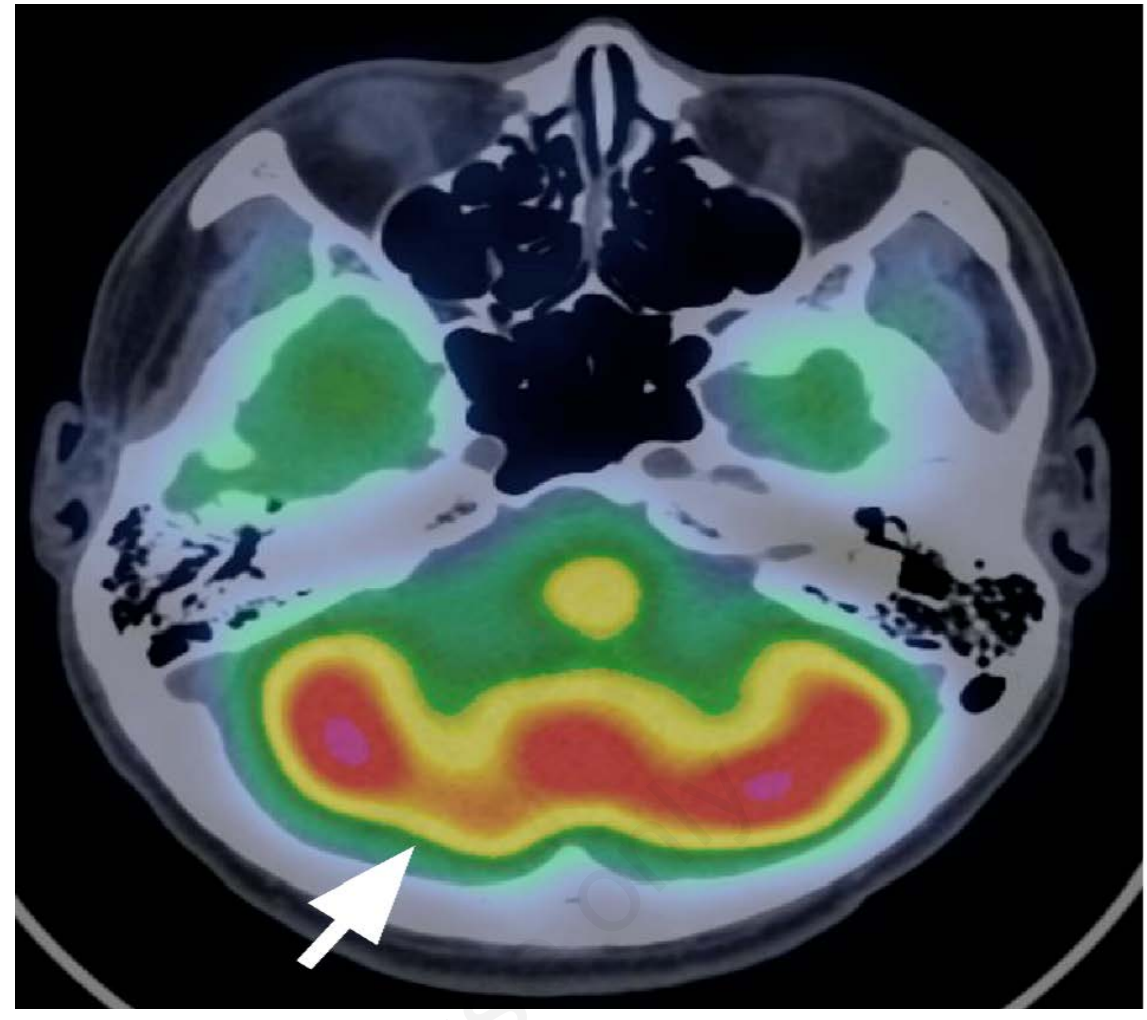

Figure 1. Tc-99m ethyl cysteinate dimer (ECD) brain perfusion single photon computed tomography fusion with CT imaging (SPECT/CT) showed asymmetry hypoperfusion (white arrow) at right cerebellum. In comparison, homogeneous red colon in the left cerebellum means normal findings of cerebellum perfusion. Brain perfusion SPECT/CT was performed 30 minutes after intravenous injection of $740 \mathrm{MBq}$ Tc-99m ECD in a room with low level of ambient light and minimal background noise. Brain perfusion SPECT detects regional cerebral blood flow and has been widely used to evaluate various neurological disorders (white arrow head).

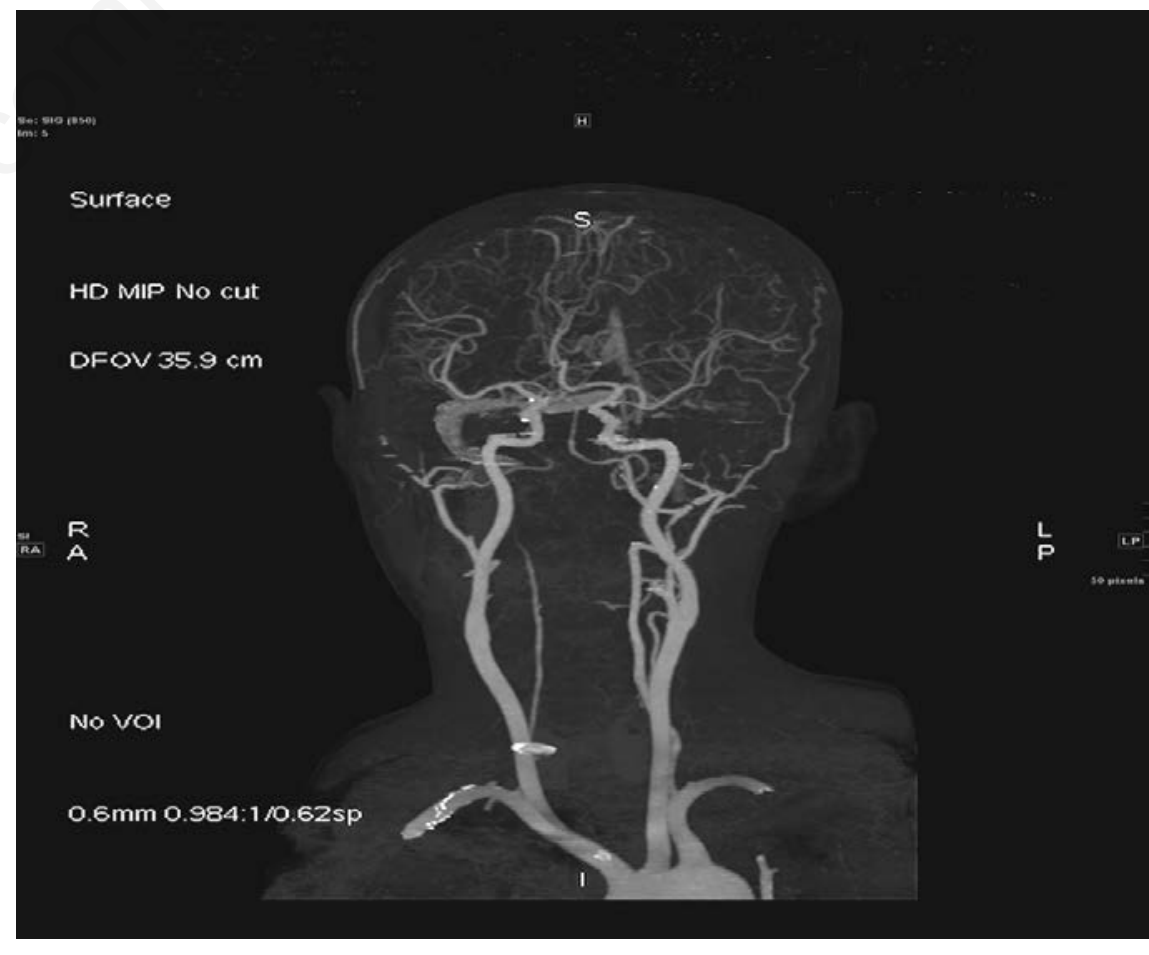

Figure 2. Cerebral computed tomographic angiography: right side vertebral artery long segmental narrowing and flow avoid from V2 to V3 (red arrow head). 
SPECT was arranged to evaluate the perfusion status which suggested hypoperfusion lesion in the right cerebellum (Figure 1) and the cerebrospinal fluid did not show significant findings, including pleocytosis or protein elevated or the presence of oligoclonal band.

During the hospitalization course, the symptoms and signs of lateral medullary stroke evolution became noticeable. The carotid echo showed extremely low flow over right vertebral artery and suggested the critical stenosis in the upstream vessels. Finally, the re-examined brain MRI with contrast and vessel wall imaging results were correlated to the thromboembolic infarction over occlusion of the posterior inferior cerebellar artery territory, which was compatible with his initial thunderclap headache presentation of the 3rd part of vertebral artery dissection.

It is important to keep in mind that differential diagnosis for orthostatic headache should not be limited to intracranial hypotension. Some patients may have inconclusive MRI or CT myelography, but there could still exist a relative CSF leak, such as CSF venous fistula or meningeal diverticula. Additionally, there have been reports on patients with orthostatic headache without CSF leak. These reports suggest that orthostatic headache can be part of the manifestation of psychiatric dis- orders, ${ }^{7}$ vestibulocochlear syndrome, ${ }^{8}$ or postural tachycardia syndrome. ${ }^{9}$

The pathophysiology behind orthostatic headache due to CSF leak is due to the loss of CSF buoyancy and downward displacement of the brain, causing traction on the sensitive nociceptors within the meninges. 4 However, the reason for this patient to present with orthostatic headache is unclear.

\section{Conclusions}

Orthostatic headache may mimic vertebral artery dissection in the initial clinical presentation. Carotid echo may be the timely and cost-effective tool for initial evaluation, and the vessel wall imaging of brain MRI could be helpful for the diagnosis if the intracranial vessels dissection is suspected. We should always keep in mind the differential diagnosis of cranial vessels dissection while thunderclap headache occurring even is related to the postural change.

\section{References}

1. Schievink WI. Spontaneous dissection of the carotid and vertebral arteries. N
Engl J Med 2001;345:467-7.

2. Lee VH, Brown RD, Mandrekar JN, Mokri B. Incidence and outcome of cervical artery dissection: a populationbased study. Neurology 2006;67:180912.

3. Mokri B, Sundt TM, Wayne $\mathrm{OH}$, Piepgras DG. Spontaneous dissection of the cervical internal carotid artery. Ann Neurol 1986;19:126-38.

4. Schievink WI. Spontaneous spinal cerebrospinal fluid leaks and intracranial hypotension. JAMA 2006;295:2286.

5. Chandra A, Suliman A, Angle N. Spontaneous dissection of the carotid and vertebral arteries: the 10-year UCSD experience. Ann Vasc Surg 2007;21:178-85.

6. Pattichis AA, Slee M. CSF hypotension: a review of its manifestations, investigation and management. J Clin Neurosci 2016;34:39-43.

7. Ferrante E, Rubino F. Orthostatic headache without intracranial hypotension: a headache due to psychiatric disorder? Headache 2014;54:1056-7.

8. Hunderfund AN, Mokri B. Orthostatic headache without CSF leak. Neurology 2008;71:1902-6.

9. Mokri B, Low PA. Orthostatic headaches without CSF leak in postural tachycardia syndrome. Neurology 2003;61:980-2. 